\title{
Sensitivity Analysis Of Vynyl Sulphone And Bis- Monochlorotriazine Reactive Groups Of Reactive Dyes
}

\author{
Sanjida Sultana \\ Primeasia University, Dhaka, Bangladesh \\ Dr. Ummul Khair Fatema \\ Bangladesh University of Textiles, Dhaka Bangladesh \\ Md. Aminul Islam \\ Huntsman Pte Ltd, Singapore
}

doi: 10.19044/esj.2016.v12n18p337 URL:http://dx.doi.org/10.19044/esj.2016.v12n18p337

\begin{abstract}
Reactive dyes contain different types of reactive groups which have different levels of sensitivity. Reactive dyeing is carried out by exhaust method on cotton knit fabric. A trichromatic combination is chosen containing vinylsulphone and bis-monochlorotriazine reactive groups. Dyeing of fabric is carried out with standard condition and variation of the conditions (electrolyte concentration, $\mathrm{pH}$, exhaustion time, fixation time and $\mathrm{M}: \mathrm{L}$ ). Colorimetric data ( $\left.\mathrm{DE}^{*}\right)$ is produced by comparing the difference between standard shade and the resulting shades by using spectrophotometer. Between the investigated reactive groups of reactive dye bismonochlorotriazine shows lower sensitivity in other terms higher robustness than vinyl sulphone.
\end{abstract}

Keywords: Sensitivity, Reactive dye, Vinylsulphone, Bismonochlorotriazine, Exhaust dyeing

\section{Introduction}

Colour of textile is one of the most important quality parameter required by the customer. But it is very difficult to achieve according to standards even with the support of modern colorimetric system and computer colour matching due to the complexity of the dyeing processes. Among all dyes reactive dyes are mostly used for dyeing cotton substrate. Reactive dyes contain different types of reactive groups which are important for dyeing because they are sensitive to different variables of dye-bath to different extent. The sensitivity causes color difference due to any change of variables (like $\mathrm{pH}$, electrolyte concentration, temperature, time, M:L, etc.). This 
sensitivity is reciprocal of robustness (correctness) where robustness is defined as the dispensing error that would produce a color difference of one unit between the correct recipe and incorrect recipe (McDonald, 1997). It follows that highly sensitive recipes are not very robust and hampers right first time to be produced. Should dyeing errors occur, the less sensitive the recipe to such errors, the more chance there is that the resultant shade will be successful (Monsoor, 2012). The goal of this work is to help the exhaust dyer to select the right recipe for higher production with lower sensitivity and higher robustness. The exhaustion and fixation of reactive dyes depends on sensitivity of reactive groups and are determined by number of factors. The most important factors are: the $\mathrm{pH}$ of dye bath, the temperature of dyeing, concentration of electrolyte, the time of dyeing and the material to liquor ratio. Among the investigated reactive groups vinyl sulphone is monofuctional and bis-MCT is bi-functional. The general structure of vinyl sulphone reactive dye is as follows:

The reactive group that has had the greatest impact on the market is the 2-sulfooxyethylsulfonyl group (Klaus, 2003). Treatment with alkali in this case causes the elimination of sulfuric acid to form a vinylsulfonyl moiety that reacts with cotton to give a dye-fiber bond. This has an elimination-addition sequence.

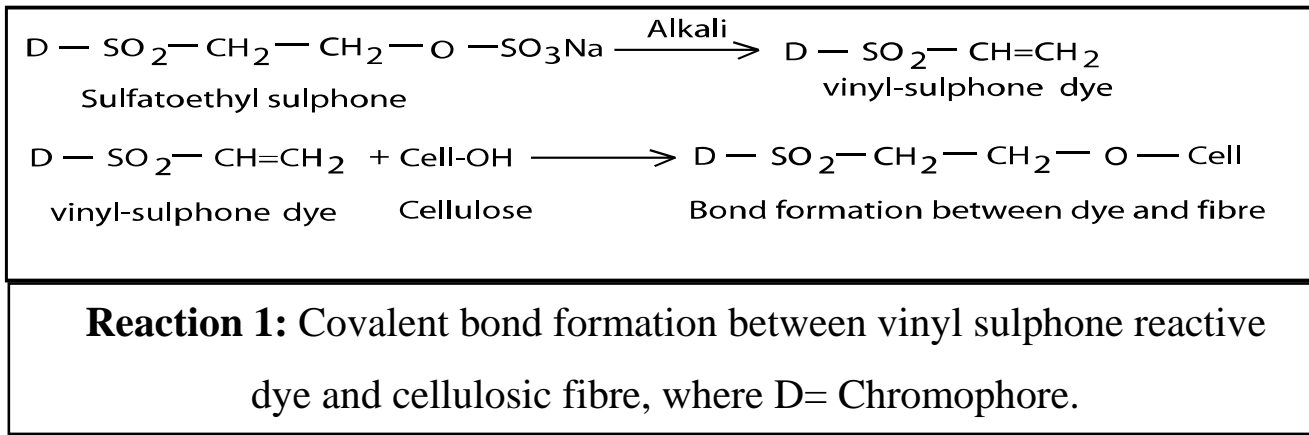

$$
\begin{gathered}
\text { Crom. }-\mathrm{SO}_{2}-\mathrm{CH}_{2}-\mathrm{CH}_{2}-\mathrm{X}+\text { Alkali } \rightarrow \text { Crom. }-\mathrm{SO}_{2}-\mathrm{CH}=\mathrm{CH}_{2}+ \\
\mathrm{HX} \\
\text { Where, } \mathrm{X}=\mathrm{OSO}_{3} \mathrm{H}, \mathrm{Cl}
\end{gathered}
$$

Reaction 2: Vinyl sulphone dye formation from 2-chloroethylsulfonyl derivatives. 
Reactive vinyl sulphones are also prepared from 2chloroethylsulfonyl derivatives, which lead to the desired intermediates by elimination of hydrogen chloride (Klaus, 2003):

Numerous derivatives of ethylsulfonyl and vinylsulfonyl groups have been prepared in recent years, though none have approached the economic importance of the sulfate esters. These dyes have medium reactivity and lower substantivity (Clariant, 2002). Exhaust dyeing temperatures between $40^{\circ} \mathrm{C}$ and $60^{\circ} \mathrm{C}$ may be chosen, depending on $\mathrm{pH}$ (John, 1995). These dyes are applicable by a wide variety of batch wise and continuous processes (John, 1995). In case of bis-MCT reactive group, two monochlorotriazine units are connected by a suitable bridge (Clariant, 2002)..

Structure 2: General structure of bis-monochlorotriazine Reactive Dye

The synthetic approach to these compounds makes it possible to combine two different chromophores in a single system, opening the way to certain color shades that are not easily accessible via a single chromophore (Klaus, 2003). Procion HEXL dyes were designed to have substantially increased substantivity, exhaustion and fixation values compared with corresponding products carrying only one chlorotriazinyl group. The triazinyl group readily lends itself to substantivity enhancement and this property, together with an application temperature of $80^{\circ} \mathrm{C}$, was used to facilitate good exhaustion and outstanding leveling properties. This approach led to improved colour utilization and in turn to less colour in the effluent (Renfrew, 1999). Reactivity of bis-MCT dye is low and the substantivity is high (Clariant, 2002). The additional technical features offer improvements in robustness and reproducibility. 


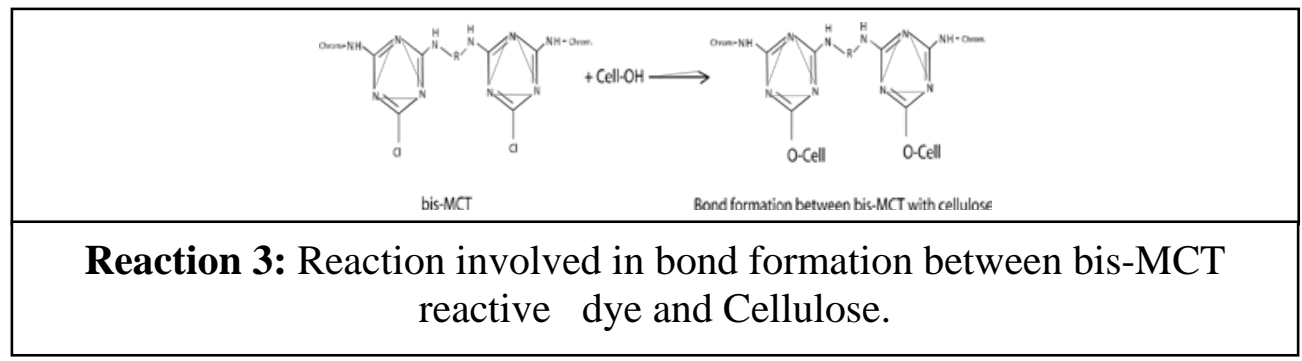

\section{Experiment:}

\section{Materials \& Method}

100\% Cotton scoured and bleached single jersey knit fabric of 180 GSM was used. Lab grade chemicals of Glauber's salt $\left(\mathrm{Na}_{2} \mathrm{SO}_{4} 10 \mathrm{H}_{2} \mathrm{O}\right)$ of $95 \%$ purity, Soda ash $\left(\mathrm{Na}_{2} \mathrm{CO}_{3}\right)$ of $98 \%$ purity, Acetic acid $\left(\mathrm{CH}_{3} \mathrm{COOH}\right)$ of 98\% purity were purchased from Merck LTd, Germany. Lab grade chemicals of Eriopon R (Sodium salt of a modified polyacrylic acid) which is a soaping agent was purchased from Huntsman Pte, Ltd. Singapore.

Table 1: Investigated reactive groups of reactive dye

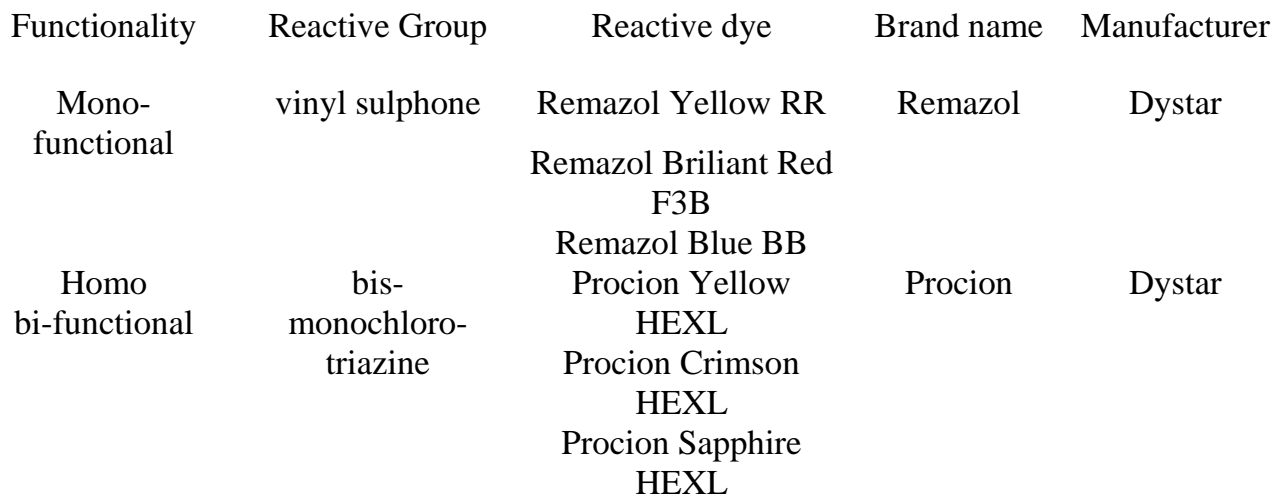

\section{Dyeing method:}

Exhaust dyeing method was used for dyeing cotton fabric. $0.3 \%$ shade was dyed for light shade and 2.1\% shade was dyed for dark shade.

Table 2: Recipe of dyeing of cotton fabric with VS reactive dye (Light shade)

\begin{tabular}{|c|c|c|c|c|c|c|c|c|c|c|}
\hline $\begin{array}{l}\text { Dyes/Che } \\
\text { micals }\end{array}$ & $\begin{array}{l}\text { Sampl } \\
\text { e } 1 \\
\text { (Stand } \\
\text { ard) }\end{array}$ & $\begin{array}{l}\text { Sam } \\
\text { ple } \\
2\end{array}$ & $\begin{array}{l}\text { Sam } \\
\text { ple3 }\end{array}$ & $\begin{array}{c}\text { Sam } \\
\text { ple } \\
4\end{array}$ & $\begin{array}{l}\text { Sam } \\
\text { ple5 }\end{array}$ & $\begin{array}{l}\text { Sam } \\
\text { ple } \\
6\end{array}$ & $\begin{array}{l}\text { Sam } \\
\text { ple7 }\end{array}$ & $\begin{array}{l}\text { Sam } \\
\text { ple8 }\end{array}$ & $\begin{array}{l}\text { Sam } \\
\text { ple9 }\end{array}$ & $\begin{array}{l}\text { Sam } \\
\text { ple } \\
10\end{array}$ \\
\hline $\begin{array}{c}\text { Remazol } \\
\text { YellowR } \\
\text { R }\end{array}$ & $\begin{array}{c}0.1 \% \\
\text { owf }\end{array}$ & $\begin{array}{c}0.1 \\
\% \\
\text { owf }\end{array}$ & $\begin{array}{c}0.1 \% \\
\text { owf }\end{array}$ & $\begin{array}{c}0.1 \\
\% \\
\text { owf }\end{array}$ & $\begin{array}{c}0.1 \% \\
\text { owf }\end{array}$ & $\begin{array}{c}0.1 \\
\% \\
\text { owf }\end{array}$ & $\begin{array}{c}0.1 \% \\
\text { owf }\end{array}$ & $\begin{array}{c}0.1 \% \\
\text { owf }\end{array}$ & $\begin{array}{c}0.1 \% \\
\text { owf }\end{array}$ & $\begin{array}{c}0.1 \\
\% \\
\text { owf }\end{array}$ \\
\hline $\begin{array}{c}\text { Remazol } \\
\text { Brilliant } \\
\text { Red F3B }\end{array}$ & $\begin{array}{l}0.1 \% \\
\text { owf }\end{array}$ & $\begin{array}{c}0.1 \\
\% \\
\text { owf }\end{array}$ & $\begin{array}{c}0.1 \% \\
\text { owf }\end{array}$ & $\begin{array}{c}0.1 \\
\% \\
\text { owf }\end{array}$ & $\begin{array}{l}0.1 \% \\
\text { owf }\end{array}$ & $\begin{array}{c}0.1 \\
\% \\
\text { owf }\end{array}$ & $\begin{array}{c}0.1 \% \\
\text { owf }\end{array}$ & $\begin{array}{c}0.1 \% \\
\text { owf }\end{array}$ & $\begin{array}{c}0.1 \% \\
\text { owf }\end{array}$ & $\begin{array}{c}0.1 \\
\% \\
\text { owf }\end{array}$ \\
\hline
\end{tabular}




\begin{tabular}{|c|c|c|c|c|c|c|c|c|c|c|c|}
\hline Remazol & $0.1 \%$ & 0.1 & $0.1 \%$ & 0.1 & $0.1 \%$ & 0.1 & $0.1 \%$ & $0.1 \%$ & $0.1 \%$ & 0.1 & 0.1 \\
\hline Blue BB & owf & $\begin{array}{c}\% \\
\text { owf }\end{array}$ & owf & $\begin{array}{c}\% \\
\text { owf }\end{array}$ & owf & $\begin{array}{c}\% \\
\text { owf }\end{array}$ & owf & owf & owf & $\begin{array}{c}\% \\
\text { owf }\end{array}$ & $\begin{array}{c}\% \\
\text { owf }\end{array}$ \\
\hline Glauber's & 20 & 16 & 24 & 20 & 20 & 20 & 20 & 20 & 20 & 20 & 20 \\
\hline Salt & g/lit & g/lit & g/lit & g/lit & g/lit & g/lit & g/lit & g/lit & g/lit & g/lit & g/lit \\
\hline Soda ash & 12 & 12 & 12 & 9.6 & $14.4 \mathrm{~g}$ & 12 & 12 & 12 & 12 & 12 & 12 \\
\hline & g/lit & g/lit & g/lit & g/lit & $/ \mathrm{lt}$ & g/lit & g/lit & g/lit & g/lit & g/lit & g/lit \\
\hline M:L & 1:8 & $1: 8$ & 1:8 & $1: 8$ & $1: 8$ & $1: 8$ & $1: 8$ & 1:8 & $1: 8$ & $1: 6$ & $1: 10$ \\
\hline $\mathrm{pH}$ & \multicolumn{11}{|c|}{$10.1-10.5$} \\
\hline Exhaustio & 30 & 30 & 30 & 30 & 30 & 15 & 45 & 30 & 30 & 30 & 30 \\
\hline n time & $\min$ & $\min$ & $\min$ & $\min$ & $\min$ & $\min$ & $\min$ & $\min$ & $\min$ & $\min$ & $\min$ \\
\hline Fixation & 60 & 60 & 60 & 60 & 60 & 60 & 60 & 40 & 80 & 60 & 60 \\
\hline time & $\min$ & $\min$ & $\min$ & $\min$ & $\min$ & $\min$ & $\min$ & $\min$ & $\min$ & $\min$ & $\min$ \\
\hline Temperat & $60^{\circ} \mathrm{C}$ & $60^{\circ}$ & $60^{\circ} \mathrm{C}$ & $60^{\circ}$ & $60^{\circ} \mathrm{C}$ & $60^{\circ}$ & $60^{\circ} \mathrm{C}$ & $60^{\circ} \mathrm{C}$ & $60^{\circ} \mathrm{C}$ & $60^{\circ}$ & $60^{\circ}$ \\
\hline ure & & $\mathrm{C}$ & & $\mathrm{C}$ & & $\mathrm{C}$ & & & & $\mathrm{C}$ & $\mathrm{C}$ \\
\hline
\end{tabular}

Table 3: Recipe of dyeing of cotton fabric with VS reactive dye (Dark shade)

\begin{tabular}{|c|c|c|c|c|c|c|c|c|c|c|c|}
\hline $\begin{array}{l}\text { Dyes/Che } \\
\text { micals }\end{array}$ & $\begin{array}{l}\text { Sampl } \\
\text { e } 1 \\
\text { (Stand } \\
\text { ard) }\end{array}$ & $\begin{array}{c}\text { Sam } \\
\text { ple } \\
2\end{array}$ & $\begin{array}{l}\text { Sam } \\
\text { ple3 }\end{array}$ & $\begin{array}{c}\text { Sam } \\
\text { ple } \\
4\end{array}$ & $\begin{array}{l}\text { Sam } \\
\text { ple5 }\end{array}$ & $\begin{array}{c}\text { Sam } \\
\text { ple } \\
6\end{array}$ & $\begin{array}{l}\text { Sam } \\
\text { ple7 }\end{array}$ & $\begin{array}{l}\text { Sam } \\
\text { ple8 }\end{array}$ & $\begin{array}{l}\text { Sam } \\
\text { ple9 }\end{array}$ & $\begin{array}{c}\text { Sam } \\
\text { ple } \\
10\end{array}$ & $\begin{array}{c}\text { Sam } \\
\text { ple } \\
11\end{array}$ \\
\hline Remazol & $0.7 \%$ & 0.7 & $0.7 \%$ & 0.7 & $0.7 \%$ & 0.7 & $0.7 \%$ & $0.7 \%$ & $0.7 \%$ & 0.7 & 0.7 \\
\hline $\begin{array}{c}\text { YellowR } \\
\text { R }\end{array}$ & owf & $\begin{array}{l}\% \\
\text { wf }\end{array}$ & owf & $\begin{array}{c}\% \\
\text { owf }\end{array}$ & owf & $\begin{array}{c}\% \\
\text { owf }\end{array}$ & owf & owf & owf & $\begin{array}{c}\% \\
\text { owf }\end{array}$ & $\begin{array}{c}\% \\
\text { owf }\end{array}$ \\
\hline Remazol & $0.7 \%$ & 0.7 & $0.7 \%$ & 0.7 & $0.7 \%$ & 0.7 & $0.7 \%$ & $0.7 \%$ & $0.7 \%$ & 0.7 & 0.7 \\
\hline $\begin{array}{l}\text { Brilliant } \\
\text { Red F3B }\end{array}$ & owf & $\begin{array}{c}\% \\
\text { owf }\end{array}$ & owf & $\begin{array}{c}\% \\
\text { owf }\end{array}$ & owf & $\begin{array}{c}\% \\
\text { owf }\end{array}$ & owf & owf & owf & $\begin{array}{c}\% \\
\text { owf }\end{array}$ & $\begin{array}{c}\% \\
\text { owf }\end{array}$ \\
\hline Remazol & $0.7 \%$ & 0.7 & $0.7 \%$ & 0.7 & $0.7 \%$ & 0.7 & $0.7 \%$ & $0.7 \%$ & $0.7 \%$ & 0.7 & 0.7 \\
\hline Blue BB & owf & $\begin{array}{c}\% \\
\text { owf }\end{array}$ & owf & $\begin{array}{c}\% \\
\text { owf }\end{array}$ & owf & $\begin{array}{c}\% \\
\text { owf }\end{array}$ & owf & owf & owf & $\begin{array}{c}\% \\
\text { owf }\end{array}$ & $\begin{array}{c}\% \\
\text { owf }\end{array}$ \\
\hline $\begin{array}{l}\text { Glauber's } \\
\text { Salt }\end{array}$ & $\begin{array}{l}50 \\
\text { g/lit }\end{array}$ & $\begin{array}{c}40 \\
\text { g/lit }\end{array}$ & $\begin{array}{c}60 \\
\text { g/lit }\end{array}$ & $\begin{array}{c}50 \\
\text { g/lit }\end{array}$ & $\begin{array}{l}50 \\
\text { g/lit }\end{array}$ & $\begin{array}{l}50 \\
\text { g/lit }\end{array}$ & $\begin{array}{l}50 \\
\text { g/lit }\end{array}$ & $\begin{array}{l}50 \\
\text { g/lit }\end{array}$ & $\begin{array}{l}50 \\
\text { g/lit }\end{array}$ & $\begin{array}{l}50 \\
\text { g/lit }\end{array}$ & $\begin{array}{c}50 \\
\text { g/lit }\end{array}$ \\
\hline Soda ash & $\begin{array}{c}16 \\
\text { g/lit }\end{array}$ & $\begin{array}{c}16 \\
\text { g/lit }\end{array}$ & $\begin{array}{c}16 \\
\text { g/lit }\end{array}$ & $\begin{array}{l}12.8 \\
\text { g/lit }\end{array}$ & $\begin{array}{c}19.2 \mathrm{~g} \\
/ \mathrm{lt}\end{array}$ & $\begin{array}{c}16 \\
\text { g/lit }\end{array}$ & $\begin{array}{c}16 \\
\text { g/lit }\end{array}$ & $\begin{array}{c}16 \\
\text { g/lit }\end{array}$ & $\begin{array}{c}16 \\
\text { g/lit }\end{array}$ & $\begin{array}{c}16 \\
\text { g/lit }\end{array}$ & $\begin{array}{c}16 \\
\text { g/lit }\end{array}$ \\
\hline M:L & $1: 8$ & $1: 8$ & $1: 8$ & $1: 8$ & $1: 8$ & $1: 8$ & $1: 8$ & $1: 8$ & $1: 8$ & $1: 6$ & $1: 10$ \\
\hline $\mathrm{pH}$ & & & & & & $0.6-11$ & & & & & \\
\hline $\begin{array}{l}\text { Exhaustio } \\
\text { n time } \\
\text { Fixation } \\
\text { time } \\
\text { Temperat } \\
\text { ure } \\
\end{array}$ & $\begin{array}{c}30 \\
\min \\
60 \\
\min \\
60^{\circ} \mathrm{C}\end{array}$ & $\begin{array}{c}30 \\
\min \\
60 \\
\min \\
60^{\circ} \\
\mathrm{C} \\
\end{array}$ & $\begin{array}{c}30 \\
\min \\
60 \\
\min \\
60^{\circ} \mathrm{C}\end{array}$ & $\begin{array}{c}30 \\
\text { min } \\
60 \\
\text { min } \\
60^{\circ} \\
\mathrm{C} \\
\end{array}$ & $\begin{array}{c}30 \\
\min \\
60 \\
\min \\
60^{\circ} \mathrm{C}\end{array}$ & $\begin{array}{c}15 \\
\min \\
60 \\
\min \\
60^{\circ} \\
\mathrm{C} \\
\end{array}$ & $\begin{array}{c}45 \\
\min \\
60 \\
\min \\
60^{\circ} \mathrm{C}\end{array}$ & $\begin{array}{c}30 \\
\text { min } \\
40 \\
\min \\
60^{\circ} \mathrm{C}\end{array}$ & $\begin{array}{c}30 \\
\text { min } \\
80 \\
\min \\
60^{\circ} \mathrm{C}\end{array}$ & $\begin{array}{c}30 \\
\min \\
60 \\
\min \\
60^{\circ} \\
\mathrm{C} \\
\end{array}$ & $\begin{array}{c}30 \\
\min \\
60 \\
\min \\
60^{\circ} \\
\mathrm{C} \\
\end{array}$ \\
\hline
\end{tabular}


Table 4: Recipe of dyeing of cotton fabric with bis-MCT reactive dye (Light shade)

\begin{tabular}{|c|c|c|c|c|c|c|c|c|c|c|c|}
\hline $\begin{array}{l}\text { Dyes/Che } \\
\text { micals }\end{array}$ & $\begin{array}{l}\text { Sampl } \\
\text { e } 1 \\
\text { (Stand } \\
\text { ard) }\end{array}$ & $\begin{array}{c}\text { Samp } \\
\text { le2 }\end{array}$ & $\begin{array}{l}\text { Sam } \\
\text { ple } 3\end{array}$ & $\begin{array}{l}\text { Sam } \\
\text { ple } 4\end{array}$ & $\begin{array}{l}\text { Sam } \\
\text { ple } 5\end{array}$ & $\begin{array}{l}\text { Sam } \\
\text { ple } 6\end{array}$ & $\begin{array}{l}\text { Sam } \\
\text { ple } 7\end{array}$ & $\begin{array}{l}\text { Sam } \\
\text { ple } 8\end{array}$ & $\begin{array}{l}\text { Sam } \\
\text { ple } 9\end{array}$ & $\begin{array}{c}\text { Sam } \\
\text { ple } \\
10\end{array}$ & $\begin{array}{c}\text { Sam } \\
\text { ple } \\
11\end{array}$ \\
\hline Procion & $0.1 \%$ & $0.1 \%$ & 0.1 & 0.1 & $0.1 \%$ & 0.1 & 0.1 & 0.1 & 0.1 & 0.1 & 0.1 \\
\hline Yellow & owf & owf & $\%$ & $\%$ & owf & $\%$ & $\%$ & $\%$ & $\%$ & $\%$ & $\%$ \\
\hline HEXL & & & owf & owf & & owf & owf & owf & owf & owf & owf \\
\hline Procion & $0.1 \%$ & $0.1 \%$ & 0.1 & 0.1 & $0.1 \%$ & 0.1 & 0.1 & 0.1 & 0.1 & 0.1 & 0.1 \\
\hline $\begin{array}{c}\text { Crimson } \\
\text { HEXL }\end{array}$ & owf & owf & $\begin{array}{l}\% \\
\text { owf }\end{array}$ & $\begin{array}{l}\% \\
\text { owf }\end{array}$ & owf & $\begin{array}{l}\% \\
\text { owf }\end{array}$ & $\begin{array}{l}\% \\
\text { owf }\end{array}$ & $\begin{array}{c}\% \\
\text { owf }\end{array}$ & $\begin{array}{c}\% \\
\text { owf }\end{array}$ & $\begin{array}{c}\% \\
\text { owf }\end{array}$ & $\begin{array}{c}\% \\
\text { owf }\end{array}$ \\
\hline Procion & $0.1 \%$ & $0.1 \%$ & 0.1 & 0.1 & $0.1 \%$ & 0.1 & 0.1 & 0.1 & 0.1 & 0.1 & 0.1 \\
\hline $\begin{array}{c}\text { Sapphire } \\
\text { HEXL }\end{array}$ & owf & owf & $\begin{array}{c}\% \\
\text { owf }\end{array}$ & $\begin{array}{c}\% \\
\text { owf }\end{array}$ & owf & $\begin{array}{c}\% \\
\text { owf }\end{array}$ & $\begin{array}{c}\% \\
\text { owf }\end{array}$ & $\begin{array}{c}\% \\
\text { owf }\end{array}$ & $\begin{array}{c}\% \\
\text { owf }\end{array}$ & $\begin{array}{c}\% \\
\text { owf }\end{array}$ & $\begin{array}{c}\% \\
\text { owf }\end{array}$ \\
\hline $\begin{array}{l}\text { Glauber's } \\
\text { Salt }\end{array}$ & $\begin{array}{l}20 \\
\text { g/lit }\end{array}$ & $\begin{array}{l}16 \\
\text { g/lit }\end{array}$ & $\begin{array}{c}24 \\
\text { g/lit }\end{array}$ & $\begin{array}{c}20 \\
\text { g/lit }\end{array}$ & $\begin{array}{l}20 \\
\text { g/lit }\end{array}$ & $\begin{array}{c}20 \\
\text { g/lit }\end{array}$ & $\begin{array}{c}20 \\
\text { g/lit }\end{array}$ & $\begin{array}{c}20 \\
\text { g/lit }\end{array}$ & $\begin{array}{c}20 \\
\text { g/lit }\end{array}$ & $\begin{array}{l}20 \\
\text { g/lit }\end{array}$ & $\begin{array}{c}20 \\
\text { g/lit }\end{array}$ \\
\hline Soda ash & $\begin{array}{c}12 \\
\text { g/lit }\end{array}$ & $\begin{array}{l}12 \\
\text { g/lit }\end{array}$ & $\begin{array}{c}12 \\
\text { g/lit }\end{array}$ & $\begin{array}{l}9.6 \\
\text { g/lit }\end{array}$ & $\begin{array}{c}\text { 14.4g } \\
\text { /lit }\end{array}$ & $\begin{array}{c}12 \\
\mathrm{~g} / \mathrm{lit}\end{array}$ & $\begin{array}{c}12 \\
\mathrm{~g} / \mathrm{lit}\end{array}$ & $\begin{array}{c}12 \\
\text { g/lit }\end{array}$ & $\begin{array}{c}12 \\
\text { g/lit }\end{array}$ & $\begin{array}{l}12 \\
\text { g/lit }\end{array}$ & $\begin{array}{c}12 \\
\text { g/lit }\end{array}$ \\
\hline $\begin{array}{c}\mathrm{M}: \mathrm{L} \\
\mathrm{pH}\end{array}$ & \multicolumn{11}{|c|}{$10.1-10.5$} \\
\hline $\begin{array}{l}\text { Exhaustio } \\
\text { n time }\end{array}$ & $30 \mathrm{~min}$ & $\begin{array}{c}30 \\
\min \end{array}$ & $\begin{array}{c}30 \\
\min \end{array}$ & $\begin{array}{l}30 \\
\min \end{array}$ & $\begin{array}{c}30 \\
\min \end{array}$ & $\begin{array}{c}15 \\
\min \end{array}$ & $\begin{array}{c}45 \\
\min \end{array}$ & $\begin{array}{c}30 \\
\min \end{array}$ & $\begin{array}{c}30 \\
\min \end{array}$ & $\begin{array}{c}30 \\
\text { min }\end{array}$ & $\begin{array}{c}30 \\
\text { min }\end{array}$ \\
\hline $\begin{array}{l}\text { Fixation } \\
\text { time }\end{array}$ & $60 \mathrm{~min}$ & $\begin{array}{c}60 \\
\mathrm{~min}\end{array}$ & $\begin{array}{c}60 \\
\min \end{array}$ & $\begin{array}{c}60 \\
\min \end{array}$ & $\begin{array}{c}60 \\
\text { min }\end{array}$ & $\begin{array}{c}60 \\
\min \end{array}$ & $\begin{array}{c}60 \\
\min \end{array}$ & $\begin{array}{c}40 \\
\min \end{array}$ & $\begin{array}{c}80 \\
\min \end{array}$ & $\begin{array}{c}60 \\
\text { min }\end{array}$ & $\begin{array}{c}60 \\
\text { min }\end{array}$ \\
\hline $\begin{array}{l}\text { Temperatu } \\
\text { re }\end{array}$ & $80^{\circ} \mathrm{C}$ & $80^{\circ} \mathrm{C}$ & $\begin{array}{c}80^{\circ} \\
\mathrm{C}\end{array}$ & $\begin{array}{c}80^{\circ} \\
\mathrm{C}\end{array}$ & $80^{\circ} \mathrm{C}$ & $\begin{array}{c}80^{\circ} \\
\mathrm{C}\end{array}$ & $\begin{array}{c}80^{\circ} \\
\mathrm{C}\end{array}$ & $\begin{array}{c}80^{\circ} \\
\mathrm{C}\end{array}$ & $\begin{array}{c}80^{\circ} \\
\mathrm{C}\end{array}$ & $\begin{array}{c}80^{\circ} \\
\mathrm{C}\end{array}$ & $\begin{array}{c}80^{\circ} \\
\mathrm{C}\end{array}$ \\
\hline
\end{tabular}

\begin{tabular}{|c|c|c|c|c|c|c|c|c|c|c|c|}
\hline $\begin{array}{l}\text { Dyes/Che } \\
\text { micals }\end{array}$ & $\begin{array}{c}\text { Sampl } \\
\text { e } 1 \\
\text { (Stand } \\
\text { ard) }\end{array}$ & $\begin{array}{l}\text { Sam } \\
\text { ple } 2\end{array}$ & $\begin{array}{l}\text { Sam } \\
\text { ple } 3\end{array}$ & $\begin{array}{c}\text { Samp } \\
\text { le } 4\end{array}$ & $\begin{array}{l}\text { Samp } \\
\text { le } 5\end{array}$ & $\begin{array}{l}\text { Sam } \\
\text { ple } 6\end{array}$ & $\begin{array}{l}\text { Sam } \\
\text { ple } 7\end{array}$ & $\begin{array}{l}\text { Sam } \\
\text { ple } 8\end{array}$ & $\begin{array}{l}\text { Sam } \\
\text { ple } 9\end{array}$ & $\begin{array}{c}\text { Sam } \\
\text { ple } \\
10\end{array}$ & $\begin{array}{c}\text { Sam } \\
\text { ple } \\
11\end{array}$ \\
\hline Procion & $0.7 \%$ & 0.7 & 0.7 & $0.7 \%$ & $0.7 \%$ & 0.7 & 0.7 & 0.7 & 0.7 & 0.7 & 0.7 \\
\hline Yellow & owf & $\%$ & $\%$ & owf & owf & $\%$ & $\%$ & $\%$ & $\%$ & $\%$ & $\%$ \\
\hline HEXL & & owf & owf & & & owf & owf & owf & owf & owf & owf \\
\hline Procion & $0.7 \%$ & 0.7 & 0.7 & $0.7 \%$ & $0.7 \%$ & 0.7 & 0.7 & 0.7 & 0.7 & 0.7 & 0.7 \\
\hline $\begin{array}{c}\text { Crimson } \\
\text { HEXL }\end{array}$ & owf & $\begin{array}{c}\% \\
\text { owf }\end{array}$ & $\begin{array}{c}\% \\
\text { owf }\end{array}$ & owf & owf & $\begin{array}{c}\% \\
\text { owf }\end{array}$ & $\begin{array}{l}\% \\
\text { owf }\end{array}$ & $\begin{array}{c}\% \\
\text { owf }\end{array}$ & $\begin{array}{c}\% \\
\text { owf }\end{array}$ & $\begin{array}{c}\% \\
\text { owf }\end{array}$ & $\begin{array}{c}\% \\
\text { owf }\end{array}$ \\
\hline Procion & $0.7 \%$ & 0.7 & 0.7 & $0.7 \%$ & $0.7 \%$ & 0.7 & 0.7 & 0.7 & 0.7 & 0.7 & 0.7 \\
\hline $\begin{array}{c}\text { Sapphire } \\
\text { HEXL }\end{array}$ & owf & $\begin{array}{c}\% \\
\text { owf }\end{array}$ & $\begin{array}{c}\% \\
\text { owf }\end{array}$ & owf & owf & $\begin{array}{c}\% \\
\text { owf }\end{array}$ & $\begin{array}{l}\% \\
\text { owf }\end{array}$ & $\begin{array}{c}\% \\
\text { owf }\end{array}$ & $\begin{array}{c}\% \\
\text { owf }\end{array}$ & $\begin{array}{c}\% \\
\text { owf }\end{array}$ & $\begin{array}{c}\% \\
\text { owf }\end{array}$ \\
\hline $\begin{array}{l}\text { Glauber’s } \\
\text { Salt }\end{array}$ & $\begin{array}{l}50 \\
\text { g/lit }\end{array}$ & $\begin{array}{c}40 \\
\sigma / l i t\end{array}$ & $\begin{array}{l}60 \\
\text { g/lit }\end{array}$ & $\begin{array}{l}50 \\
g / l i t\end{array}$ & $\begin{array}{c}50 \\
\text { g/lit }\end{array}$ & 50 & 50 & 50 & 50 & 50 & 50 \\
\hline Soda ash & $\begin{array}{l}16 \\
\text { g/lit }\end{array}$ & $\begin{array}{l}16 \\
\text { g/lit }\end{array}$ & $\begin{array}{l}16 \\
g / l i t\end{array}$ & $\begin{array}{c}\text { 12.8g } \\
\text { lit }\end{array}$ & $\begin{array}{c}19.2 \mathrm{~g} \\
\text { /lit }\end{array}$ & $\begin{array}{l}16 \\
\text { g/lit }\end{array}$ & $\begin{array}{l}16 \\
\text { g/lit }\end{array}$ & $\begin{array}{l}16 \\
\text { g/lit }\end{array}$ & $\begin{array}{l}16 \\
\text { g/lit }\end{array}$ & $\begin{array}{l}16 \\
\text { g/lit }\end{array}$ & $\begin{array}{l}16 \\
\text { g/lit }\end{array}$ \\
\hline M:L & 1:8 & $1: 8$ & $1: 8$ & 1:8 & $1: 8$ & $1: 8$ & $1: 8$ & $1: 8$ & $1: 8$ & $1: 6$ & $1: 10$ \\
\hline $\mathrm{pH}$ & \multicolumn{11}{|c|}{$10.6-11$} \\
\hline $\begin{array}{l}\text { Exhaustio } \\
\text { n time }\end{array}$ & $30 \mathrm{~min}$ & $\begin{array}{c}30 \\
\min \end{array}$ & $\begin{array}{c}30 \\
\min \end{array}$ & $\begin{array}{c}30 \\
\min \end{array}$ & $\begin{array}{l}30 \\
\min \end{array}$ & $\begin{array}{c}15 \\
\min \end{array}$ & $\begin{array}{c}45 \\
\min \end{array}$ & $\begin{array}{c}30 \\
\min \end{array}$ & $\begin{array}{c}30 \\
\min \end{array}$ & $\begin{array}{l}30 \\
\min \end{array}$ & $\begin{array}{l}30 \\
\min \end{array}$ \\
\hline $\begin{array}{c}\text { Fixation } \\
\text { time }\end{array}$ & $60 \mathrm{~min}$ & $\begin{array}{c}60 \\
\min \end{array}$ & $\begin{array}{c}60 \\
\min \end{array}$ & $\begin{array}{c}60 \\
\mathrm{~min}\end{array}$ & $\begin{array}{c}60 \\
\min \end{array}$ & $\begin{array}{c}60 \\
\min \end{array}$ & $\begin{array}{c}60 \\
\min \end{array}$ & $\begin{array}{l}40 \\
\min \end{array}$ & $\begin{array}{c}80 \\
\min \end{array}$ & $\begin{array}{c}60 \\
\min \end{array}$ & $\begin{array}{c}60 \\
\min \end{array}$ \\
\hline $\begin{array}{l}\text { Temperatu } \\
\text { re }\end{array}$ & $80^{\circ} \mathrm{C}$ & $\begin{array}{c}80^{\circ} \\
\mathrm{C} \\
\end{array}$ & $\begin{array}{c}80^{\circ} \\
\mathrm{C}\end{array}$ & $80^{\circ} \mathrm{C}$ & $80^{\circ} \mathrm{C}$ & $\begin{array}{c}80^{\circ} \\
\mathrm{C}\end{array}$ & $\begin{array}{c}80^{\circ} \\
\mathrm{C}\end{array}$ & $\begin{array}{c}80^{\circ} \\
\mathrm{C} \\
\end{array}$ & $\begin{array}{c}80^{\circ} \\
\mathrm{C} \\
\end{array}$ & $\begin{array}{c}80^{\circ} \\
\mathrm{C} \\
\end{array}$ & $\begin{array}{c}80^{\circ} \\
\mathrm{C}\end{array}$ \\
\hline
\end{tabular}


Working Procedure:

$1 \%$ stock solution of each dye has been prepared carefully.

Additional water has been calculated and taken in dyeing pots.

Accurate amount of dye solution one by one has been pipette in every

pot.

$>\quad$ Pre-treated fabrics loaded in those pots.

$>\quad$ Dyeing pots are loaded into the dyeing machine by setting exhaustion temperature for listed time.

$>\quad$ Addition of soda ash after maintaining required exhaustion time and checking $\mathrm{pH}$ of dyeing solution has been done and recorded.

$>\quad$ Then dyeing continued at mentioned temperature for required time.

After-treatment:

$>\quad$ The dyed samples were rinsed in cold water for 5 minutes.

$>\quad$ The labdip neutralized in $1 \mathrm{gm} / \mathrm{lit}$ acetic acid at $40^{\circ} \mathrm{C}$ for 10 minutes.

$>\quad$ The labdip treated in $1 \mathrm{gm} /$ lit soaping agent (Eriopon $\mathrm{R}$ ) at $90^{\circ} \mathrm{C}$ for

10 minutes.

$>\quad$ The labdip rinsed in hot water at $80^{\circ} \mathrm{C}$ for 10 minutes.

$>\quad$ The labdip was cold rinsed for 5 minutes.

\section{Results \& Discussion}

For measuring color difference Datacolor $600^{T M}$ Spectrophotometer (made in: USA) have been used which is a reflectance spectrophotometer. For convenience of result discussion a grading system for sensitivity has been introduced.

$$
\begin{gathered}
\text { DE* value } \\
0-0.5 \\
>0.5-1
\end{gathered}
$$

Table 6: Grading system

$$
\begin{aligned}
& \text { Sensitivity } \\
& \text { Low } \\
& \text { Medium } \\
& \text { High }
\end{aligned}
$$

\begin{tabular}{ccccc}
\hline \multicolumn{5}{c}{ Table 7: Color difference $\left(\mathrm{DE}^{*}\right)$ of VS and BIS-MCT reactive dye (Light shade) } \\
Criteria & DE* of & Sensitivity of & DE* of Bis- & Sensitivity of \\
& VS & VS & MCT & Bis-MCT \\
Low Salt & 0.58 & Medium & 0.69 & Medium \\
More Salt & 1.22 & High & 0.29 & Low \\
Low Alkali & 0.74 & Medium & 0.3 & Low \\
High Alkali & 1.29 & High & 0.5 & Low \\
Low Exhaustion Time & 0.67 & Medium & 0.16 & Low \\
High Exhaustion Time & 0.73 & Medium & 0.35 & Low \\
Low Fixation Time & 0.52 & Medium & 0.64 & Medium \\
High Fixation Time & 1.08 & High & 0.99 & Medium \\
Low M:L & 1.02 & High & 0.21 & Low \\
High M:L & 0.32 & Low & 0.26 & Low \\
\hline
\end{tabular}


From table no. 7 it can be said that VS reactive group shows higher sensitivity to electrolyte concentration variation, higher sensitivity to $\mathrm{pH}$ variation, medium sensitivity to exhaustion time variation, higher sensitivity to fixation time variation and higher sensitivity to M:L variation. It can also be said that Bis-MCT reactive group shows medium sensitivity to electrolyte concentration variation, low sensitivity to $\mathrm{pH}$ variation, low sensitivity to exhaustion time variation, medium sensitivity to fixation time variation and low sensitivity to $\mathrm{M}: \mathrm{L}$ variation.

Table 8: Color difference of VS and BIS-MCT reactive groups of reactive dye

\begin{tabular}{ccccc} 
Criteria & VS & $\begin{array}{c}\text { (Dark shade). } \\
\text { Sensitivity of }\end{array}$ & Bis-MCT & $\begin{array}{c}\text { Sensitivity of } \\
\text { Bis-MCT }\end{array}$ \\
Low Salt & 0.58 & Medium & 1.02 & High \\
More Salt & 0.76 & Medium & 1.24 & High \\
Low Alkali & 0.36 & Low & 0.49 & Low \\
High Alkali & 0.46 & Low & 1.06 & High \\
Low Exhaustion Time & 0.03 & Low & 0.81 & High \\
High Exhaustion Time & 0.34 & Low & 1.04 & High \\
Low Fixation Time & 1.3 & High & 0.35 & Low \\
High Fixation Time & 0.34 & Low & 0.53 & Medium \\
Low M:L & 0.49 & Low & 0.74 & Medium \\
High M:L & 0.39 & Low & 0.63 & Medium \\
\hline
\end{tabular}

From table no. 8 it can be said that VS reactive group shows medium sensitivity to electrolyte concentration variation, low sensitivity to $\mathrm{pH}$ variation, low sensitivity to exhaustion time variation, higher sensitivity to fixation time variation and low sensitivity to M:L variation. It can also be said that Bis-MCT reactive group shows high sensitivity to electrolyte concentration variation, high sensitivity to $\mathrm{pH}$ variation, high sensitivity to exhaustion time variation, medium sensitivity to fixation time variation and medium sensitivity to M:L variation.

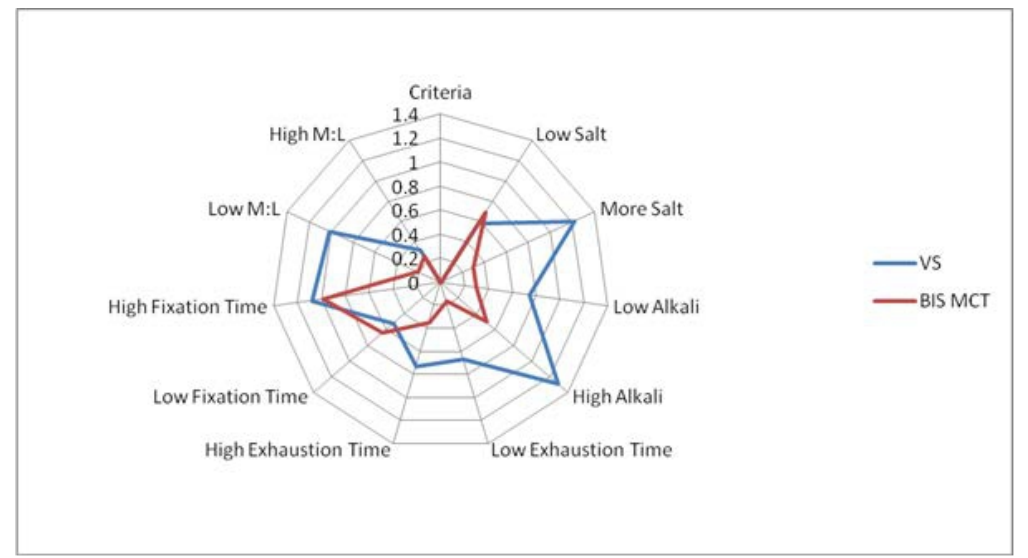

Fig 1: Comparative sensitivity of VS and Bis-MCT reactive groups of reactive dye (Light shade). 


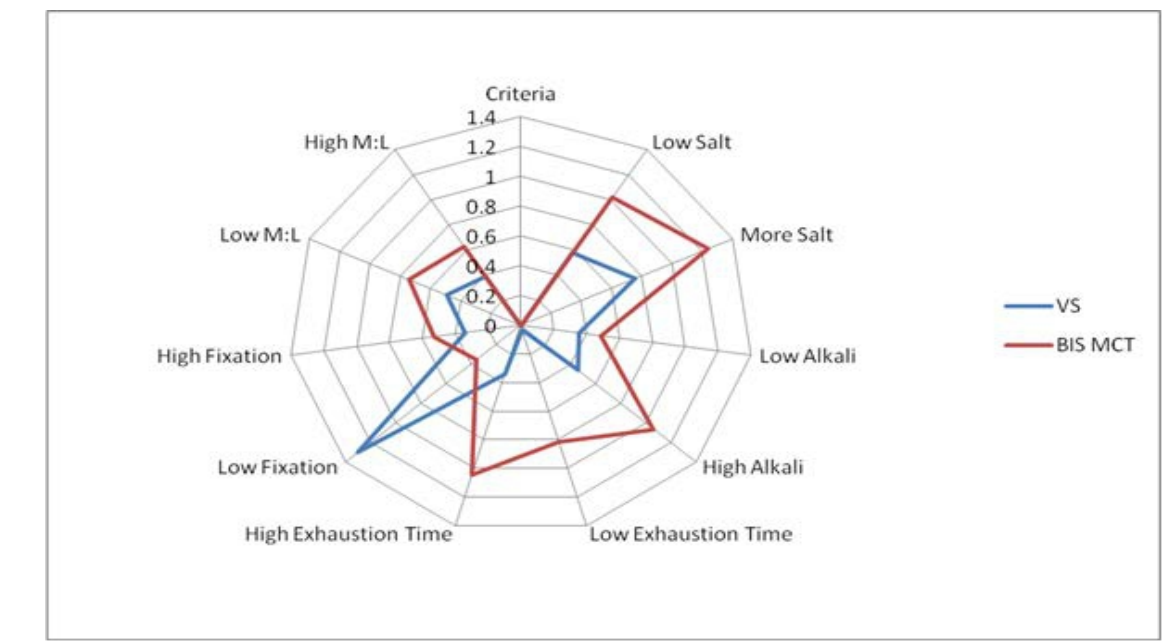

Fig 2: Comparative sensitivity of VS and Bis-MCT reactive groups of reactive dye (Dark shade).

From fig. 1 it is seen that in case of VS reactive group for Light shade salt sensitivity is high, alkali sensitivity is high, exhaustion time sensitivity is medium, fixation time sensitivity is high and M:L sensitivity is high. But for dark shade from fig. 2 salt sensitivity is medium, alkali sensitivity is low, exhaustion time sensitivity is low, fixation time sensitivity is high and M:L sensitivity is low. In all the parameters except fixation time variation vinyl sulphone dye shows improved performance with increasing shade \% i.e, electrolyte sensitivity decreased from high to medium, $\mathrm{pH}$ sensitivity decreased from high to low, exhaustion time sensitivity goes from medium to low and M:L sensitivity decreased from high to low. But the fixation time sensitivity is unchanged which means that the vinyl sulphone dye is highly fixation time sensitive.

Vinyl sulphone dyes have moderate reactivity and lower substantivity (Clariant, 2002). The substantivity can be increased by increasing the $\mathrm{pH}$ upto 11 (Tanveer, 2012). When the shade\% is high, the $\mathrm{pH}$ of the solution is also high. As a result the dye substantivity is increased with increasing $\mathrm{pH}$ and the sensitivity is decreased resulting better performance. Hence this dyestuff is suitable for dyeing medium to dark shades.

From fig. 1 it is seen that incase of BIS-MCT reactive group of reactive dye for light shade salt sensitivity is medium, alkali sensitivity is low, exhaustion time sensitivity is low, fixation time sensitivity is medium and M:L sensitivity is low. But for dark shade (fig. 2) salt sensitivity is high, alkali sensitivity is high, exhaustion time sensitivity is high, fixation time sensitivity is medium and M:L sensitivity is medium. In all the parameters except fixation time the performance have been decreased with increased shade\% i.e, salt sensitivity increases from medium to high, $\mathrm{pH}$ sensitivity increases from low to high, exhaustion time sensitivity increases from low 
to high and M:L sensitivity goes from low to medium. But only fixation time sensitivity is in same order i.e, it is fixation time sensitive of medium order.

Reactivity of bis-MCT dye is low and the substantivity is high (Clariant, 2002). The bond energy of $\mathrm{Cl}$ (Chlorine) is $77 \mathrm{kcal} / \mathrm{gm}$ which is moderate and less than F (fluorine) (Shenai, 2000). Hence dyeing temperature is higher to reduce color strike (instant pickup of dyestuffs at the fibre surface) to improve migration and levelness. $\mathrm{pH}$ is lower in light shade and higher in dark shade. So, at high temperature when $\mathrm{pH}$ is increased by increasing alkali dosage, alkaline hydrolysis occurs with the release of Chlorine $(\mathrm{Cl})$ which results low yield of depth. For this reason the performance is decreased with increase of shade depth.

\section{Conclusion}

From results and discussion it is seen that VS reactive group shows high sensitivity incase of light shade and hence this dyestuff is suitable for dyeing medium to dark shades. Bis-MCT reactive group shows high sensitivity incase of dark shade and hence this reactive group is suitable for dyeing light shade.

\section{References:}

Roderick McDonald (Editor) (1997), Color Physics for Industry, SDC, Bradford. England, p. 292-372.

Mansoor Iqbal, Zeeshen Khatri, Aleem Ahmed, Javaid Mughal, Kamran Ahmed, (2012), "Prediction of low-sensitivity reactive dye recipe in exhaust dyeing influenced by material to liquor ratio and nature of salt”, Journal of Soudi Chemical Society, January, 16, 1, p.1-6.

John Shore (editor) (1995), Cellulosic Dyeing, Society of Dyers and Colorists, p.189-245.

Klaus Hunger (editor) (2003), Industrial Dyes, Chemistry, Properties, Application, WILEY-VCH, p. 113-118.

Clariant (2002) Technical data sheet.

A Hunter M Renfrew (1999), Reactive Dyes for Textile Fibres, Society of Dyers and Colorists, p. 105-138.

Tanveer Hossain (2012), Exhaust dyeing with reactive dyes, academia.edu, p. 1-26.

Prof, V.A. Shenai (2000), Technology of Textile Processing, Chemistry of Dyes and principles of dyeing, Volume-II, p. 495-562. 